\title{
RADYOAKTIF PERTEKNETAT OKSO ANYONU ADSORPSIYONUNUN TERMODINAMIK ANALIZI
}

\author{
${ }^{1}$ Fatih DEMIR ${ }^{(\mathbb{D})},{ }^{2}$ Omer LACIN ${ }^{(\mathbb{D})},{ }^{3}$ Burak BASTABAN \\ Atatürk Üniversitesi, Mühendislik Fakültesi, Kimya Mühendisliği Bölümü, Erzurum, TÜRKIYYE \\ 2olacin@atauni.edu.tr
}

(Geliş/Received: 04.03.2019; Kabul/Accepted in Revised Form: 13.06.2019)

ÖZ: Kesikli sistemde çalışılan adsorpsiyon tekniği ile aktif karbon üzerine radyoaktif perteknetat okso

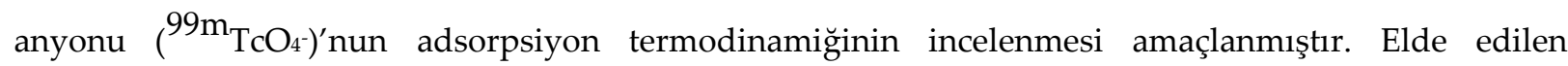
verilerden faydalanarak termodinamik parametreler; $\Delta \mathrm{H}^{\mathrm{o}}=0,346 \mathrm{~kJ} \cdot \mathrm{mol}^{-1}, \Delta \mathrm{S}^{\mathrm{o}}=0,029 \mathrm{~kJ} \cdot \mathrm{mol}^{-1}, \Delta \mathrm{G}^{\mathrm{o}}=-7,92$ $(288 \mathrm{~K})$ ile $-8,78(318 \mathrm{~K}) \mathrm{kJ} \cdot \mathrm{mol}^{-1}, \Delta \mathrm{Hx}=0,28 \mathrm{~kJ} \cdot \mathrm{mol}^{-1} \mathrm{ve}$ standart serbest enerji değerinin sıfır olduğu sıcaklık $12 \mathrm{~K}$ olarak hesaplanmıştır. Bu bulgular sonucunda adsorpsiyon prosesinin; fiziksel ve endotermik olarak gerçekleştiği ve 12 K'in üzerindeki her sıcaklıkta adsorpsiyonun uygun ve kendiliğinden gerçekleşeceği bulguları elde edilmiştir. Sonuç olarak, belirli bir limitin üzerine çıkıldığında kansere neden olan radyoaktif teknesyumun aktif karbon üzerine adsorpsiyon termodinamik analizinin incelenmesi sonucu elde edilen bulguların endüstriyel çapta kurulacak bir tesis dizaynına katkıda bulunacağı söylenebilir.

Anahtar Kelimeler: Radyoaktif madde, Aktif Karbon, Termodinamik, Adsorpsiyon

\section{Thermodynamic Analysis of Radioactive Pertechnetate Oxo Anion Adsorption}

\begin{abstract}
The aim of this study is to investigate thermodynamics of radioactive pertechnetate okso anion ${ }^{99 \mathrm{mTCO}_{4}}$ ) ) over the active carbon using the adsorption technique in batched systems. According to the obtained results thermodynamic parameters are calculated as $\Delta \mathrm{H}^{\circ}=0.346 \mathrm{~kJ} \cdot \mathrm{mol}^{-1}, \Delta \mathrm{S}^{\mathrm{o}}=0.029 \mathrm{~kJ} \cdot \mathrm{mol}^{-1}$, $\Delta \mathrm{G}^{\mathrm{o}}=-7.92\left(15^{\circ} \mathrm{C}\right)$ ile $-8.78\left(45^{\circ} \mathrm{C}\right) \mathrm{kJ} \cdot \mathrm{mol}^{-1}, \Delta \mathrm{Hx}=0,28 \mathrm{~kJ} \cdot \mathrm{mol}^{-1}$ and also the temperature at which the standart free energy value equals to zero calculated as $12 \mathrm{~K}$. These results indicated that the adsorption process is physical and endothermic and at temparatures over $12 \mathrm{~K}$ adsorption will take place spontaneously and favorably. In conclusion, it can be said that the results obtained from the thermodynamic analysis of radioactive technetium (which may cause cancer if the limit is exceeded) over the active carbon can contribute to the design of an industrial facility.
\end{abstract}

Key Words: Radioactive Substance, Activated Carbon, Thermodynamic, Adsorption

\section{GİRIŞ(INTRODUCTION)}

Nükleer tıp, insan vücudundaki çeşitli biyokimyasal ve fizyolojik işlemlere katılabilecek radyoaktif bileşikler yardımıyla tanısal görüntüleme ve tedavi uygulamalarını içeren bir bilim dalıdır. Bu bilim dalında en yaygın kullanılan radyoaktif element teknesyumdur (Hercigonja ve diğ. 2012).

Dünyadaki uranyum cevherlerinde nanogram miktarlarında bulunan teknesyum (Tc), nükleer reaktörlerde $\mathrm{Mo}$, Ru ve $\mathrm{Nb}^{\prime}$ un kozmik ışın reaksiyonu sonucu yapay olarak elde edilebilir. Plütonyum239 ve uranyum-235'in termal fisyonu ile de \% 6'llk bir verimle ${ }^{99}$ Teknesyum üretilebilir (Leon ve 
dĭ̆.2005). İlk önce Perrier ve diğ. 1937'de, molibdeni, bir nötronla bombardıman ederek Tc sentezlemeyi başarmışlardır (Şekil 1) (Shia ve diğ. 2012).

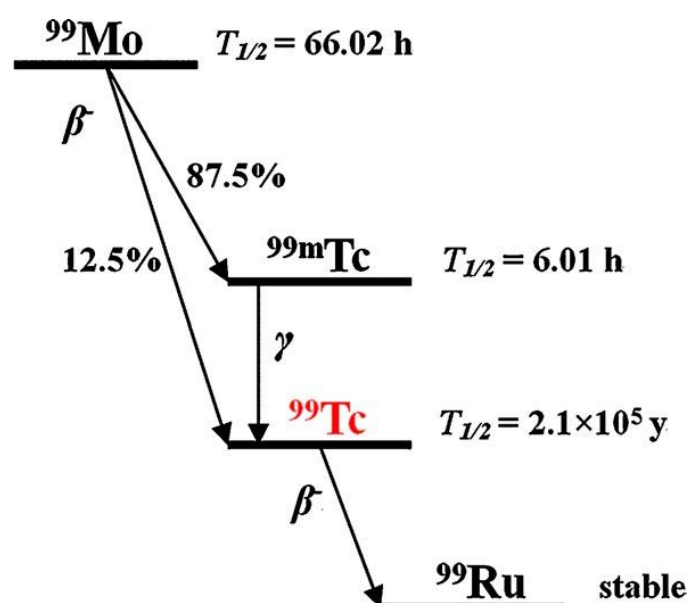

Şekil 1. ${ }^{99} \mathrm{Mo}$ ninbozunma şeması

Figure 1. Decayscheme of ${ }^{99} \mathrm{Mo}$

Şekil l'de görülebileceği gibi, Tc'nin en kararlı radyoaktif izotopu, 6 saatlik bir yarı ömre sahip ${ }^{99 m}$ Tc'dir. Bu izotop, gama ışınları yayarak ${ }^{99}$ Tc'ye bozunur. ${ }^{99} \mathrm{Tc}, 0,294 \mathrm{MeV}^{\prime}$ lik maksimum bozunma enerjisine sahip saf bir $\beta^{-}$yayıcı olduğundan dolayı, radyasyon sayımı ile ölçülebilir özelliğe sahiptir (Banavali ve diğ. 1995).

99mTc, yeraltı sularını kirleten tehlikeli nükleer atıklardan biridir (Hercigonja ve dĭg. 2012). Çünkü, 99mTc ve \% 0,9 NaCl çözeltisi karıştırıldığında, izotonik, renksiz, berrak ve steril bir sodyum perteknetat solüsyonu elde edilir. Bu solüsyon tanı için doğrudan hastaya uygulanabilir. Solüsyondaki perteknetat

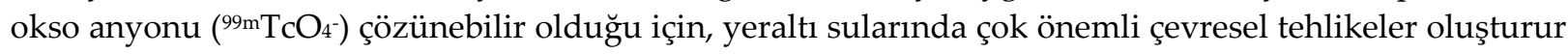
(Bishop ve diğ. 2011). ${ }^{99}$ Tc'nin immobilizasyonu uzun süreli ve kalıc1 imha işlemlerinden önce yapılmalıdır. Bu nedenle, daha büyük miktarda atık üretmeden ${ }^{99} \mathrm{Tc}{ }^{\prime}$ nin uzaklaştırılması gereklidir (Del Cul ve diğ. 1993). Birleşik Devletler Çevre Koruma Ajansı (USEPA), içme sularında yıllık maksimum 0,04 milisievert (mSv) değerinde ${ }^{99} \mathrm{Tc}$ içeriği olması gerektiğini belirlemiştir (USEPA, 2002). Bu değer aşılırsa, insanların kansere yakalanması kaçınılmaz olacağı belirtilmiştir (Del Cul ve diğ. 1993).

Radyoaktif maddelerin sulu çözeltilerden uzaklaştırılmasında; kimyasal çöktürme, buharlaştırma, çözücü özütleme, membran prosesleri ve adsorpsiyon gibi çeşitli yöntemler uygulanmıştır (Mahmoud ve diğ. 2014). Çöktürme ve çözücü özütlemenin, düşük seçiciliğe ve büyük miktarlarda katı çamur oluşumuna sebep olduğu, (Sangvanich ve diğ. 2010), buharlaştırma yönteminin korozyon, kireçlenme veya köpüklenme gibi sorunlara yol açtığı (Rout ve diğ. 2006) ve membran proseslerinin, yüksek maliyet ve membran tıkanması gibi kısıtlamalara sahip olduğu (Fu ve Wang, 2011) belirtilmiştir. Adsorpsiyon yönteminin ise, düşük maliyet, esneklik ve tasarım basitliği, kullanım kolaylığı ve diğer tekniklere kıyasla toksik kirleticilere duyarsızlık gibi birçok avantaja sahip olduğundan dolayı daha çok tercih edilen bir yöntem olmuştur (Mahmoud ve diğ. 2014).

Literatürde adsorpsiyon yöntemiyle, ${ }^{99} \mathrm{Tc}$ 'nin yeraltı sularından uzaklaştırılması için kullanılan bazı adsorbanlar şunlardır: alümina ve goetit (Kumar ve diğ. 2011), stibnit (Kumar ve dĭ̆. 2013), elementel demir (Liang ve diğ. 1996), pirit ve manyetit (Lieser ve Bauscher, 1988, Farrell ve diğ. 1999), organofilik bentonitler (Bors ve diğ. 1999, Sahkira ve diğ. 2011) ve çeşitli sentetik reçineler (Suzuki ve diğ. 2009, Chen ve Veltkamp, 2002).

Aktif karbon, geniş yüzey alanı ve gözenekli yapıya sahip karbonlu ürünlerin ticari adıdır. Kontrollü bir atmosfer ve sıcaklık şartlarında, yüksek sıcaklıkta ısıl ayrışma ile karbon bazlı malzemelerden üretilir. Aktif karbonların genellikle yoğunluğu yaklaşık $0,2 \mathrm{~g} \cdot \mathrm{cm}^{-3}$ den büyük ve yüzey alanı $400-1600$ $\mathrm{m}^{2} \cdot \mathrm{g}^{-1}$ arasında değişmekte olup adsorplama kapasitesi çok büyüktür (Holm ve diğ. 2000). 


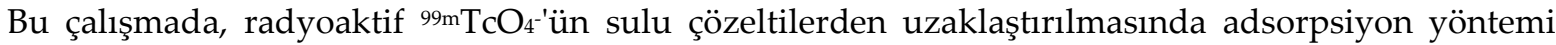
tercih edilmiş ve adsorban olarak ise aktif karbon seçilmiştir. Adsorpsiyon prosesi sonucunda elde edilen termodinamik parametreler ışığında, adsorpsiyonun doğası hakkında bilgilerin sunulmasıyla endüstriyel çapta kurulacak bir tesis dizaynına katkıda bulunacağı amaçlanmıştır.

\section{MATERYAL ve YÖNTEM(MATERIAL and METHOD)}

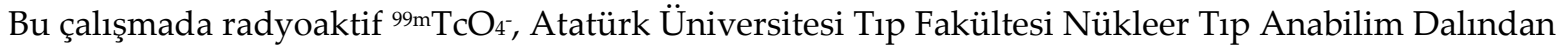

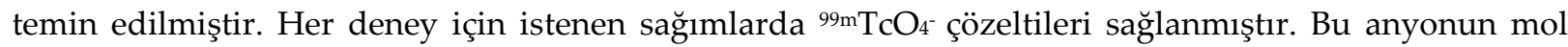
kütlesi 163 g.mol-1 olup dört hidrojen bağına sahiptir. Topolojik polar yüzey alanı $73 \times 10^{-20} \mathrm{~m}^{2}$ dir (PubChem, web).

Aktif karbon (Sigma-aldrichDarco G60) yerel bir medikal firmasından temin edilmiştir. Aktif karbonun bazı özellikleri Çizelge 1'de verilmiştir.

Çizelge 1 Aktif Karbonun Bazı Özellikleri

Table 1. Some Properties of Activated Carbon

\begin{tabular}{cllll}
\hline $\mathrm{pH}$ & $\begin{array}{l}\text { Ort. Boyut } \\
(\mathbf{m m})\end{array}$ & $\begin{array}{l}\text { Yoğunluk } \\
\left(\mathrm{g} . c m^{-3}\right)\end{array}$ & $\begin{array}{l}\text { Yüzey } \\
\text { Alanı } \\
\left(\mathbf{m}^{2} \cdot \mathbf{g}^{-1}\right)\end{array}$ & $\begin{array}{l}\text { Saflık } \\
\mathbf{\%}\end{array}$ \\
\hline $5-7$ & $0,044-0,15$ & $0,45-0,55$ & 1100 & 99 \\
\hline
\end{tabular}

Aktif karbon elenerek partikül boyutu +0,15-0,125 mm olacak şekilde seçilmiştir.

Deneyler, kesikli sistemde çalışılan adsorpsiyon tekniği ile yapılmıştır. Başlangıç konsantrasyonu, $\mathrm{pH}$, partikül boyutu, karıştırma hızı, adsorpsiyon denge süresi ve adsorban konsatrasyonu parametreleri sırasiyla; $1,0 \mathrm{mCi} . \mathrm{L}^{-1}, 8,0,+0,15-0,125 \mathrm{~mm}, 700 \mathrm{rpm}, 15$ dakika ve 2,0 g.L-1 olarak sabit tutulmuştur (Bastaban, 2014).

\section{Deneysel Yöntem(Experimental Set up)}

0,5 mCi.mL ${ }^{-1}$ 99m $\mathrm{TcO}_{4}^{-}$çözeltisinden $1 \mathrm{~mL}$ alınarak deiyonize su ile $500 \mathrm{~mL}$ 'ye tamamlanmıştır.

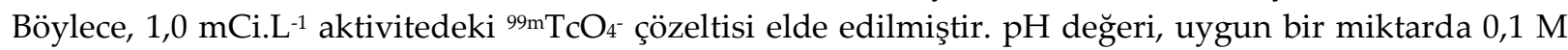
$\mathrm{NaOH}$ (sigma-aldrich, \%98) ve 0,1 M HCl (merck, \%37) çözeltileri kullanılarak dijital bir pH-metre (Thermo Orion 3-Star) yardımıyla ayarlanmıştır.

Kesikli adsorpsiyon deneyleri, 288-318 K sıcaklık aralığında $700 \mathrm{~mL}$ hacmindeki bir ceketli reaktör sisteminde sabit tutulan parametre değerleriyle gerçekleştirilmiştir. Adsorpsiyon denge süresi sonunda, nihai karışım nuçe erleniyle vakum altında süzülmüştür. Çözeltide, denge anındaki ${ }^{99 m}{ }^{2}{ }^{2}{ }^{-}{ }^{\prime}$ ün radyoaktivitesi $\left(\mathrm{C}_{\mathrm{e}}\right)$, Biodex, Atomlab 400 model dose calibrator marka dozimetre kullanılarak ölçülmüş ve Eşitlik 1'den elde edilen qe değerleri Çizelge 2' de verilmiştir.

$$
q_{e}=\frac{\left(C_{o}-C_{e}\right) V}{m}
$$

Burada $\mathrm{C}_{\mathrm{o}}$ ve $\mathrm{C}_{\mathrm{e}}\left(\mathrm{mCi} \cdot \mathrm{L}^{-1}\right)$ sırasıyla, ${ }^{99 \mathrm{~m}} \mathrm{TcO}_{4}{ }^{\prime}$ ün başlangıç ve denge radyoaktiviteleridir. $\mathrm{V}$ (L), çözelti hacmi, $\mathrm{m}(\mathrm{g})$, adsorbanın kütlesi ve $\mathrm{qe}_{\mathrm{e}}\left(\mathrm{mCi} \cdot \mathrm{g}^{-1}\right)$, adsorpsiyon kapasitesidir. 
Çizelge 2Adsropsiyonun Sıcaklıkla Değişim Verileri (konsantrasyon 1,0 mCi.L-1 99mTcO4-, pH 8,0, partikül boyutu +0,15-0,125 mm, karıştırma hızı $700 \mathrm{rpm}$, adsorpsiyon denge süresi $15 \mathrm{dk}$ ve adsorban konsatrasyonu 2,0 g.L $\left.\mathrm{L}^{-1}\right)$

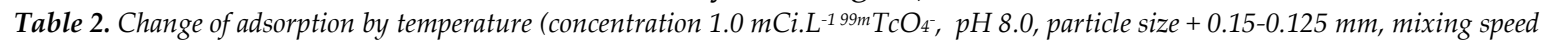

\begin{tabular}{clc}
$700 \mathrm{rpm}$, adsorption equilibrium time 15 min. and adsorbent concentration $\left.2.0 \mathrm{~g} \cdot \mathrm{L}^{-1}\right)$ \\
\hline Sicaklik $(\mathrm{K})$ & $\mathrm{Ce}_{\mathrm{e}}\left(\mathrm{mCi}_{\mathrm{L}} \mathrm{L}^{-1}\right)$ & $\mathrm{qe}\left(\mathrm{mCi} \cdot \mathrm{g}^{-1}\right)$ \\
\hline 288 & 0,320 & 0,340 \\
298 & 0,160 & 0,420 \\
308 & 0,110 & 0,445 \\
318 & 0,060 & 0,470 \\
\hline
\end{tabular}

\section{BULGULAR VE SONUÇLARIN İRDELENMESİ(RESULT AND DISCUSSION)}

\section{Adsorpsiyon Termodinamiğinin Modellenmesi(Modeling of Adsorption Thermodynamics)}

Adsorpsiyon prosesinde denge anına ulaşıldığında, hem çözeltide hem de katı yüzeyinde adsorplanan maddenin konsantrasyonunda bir değişim gözlemlenmez. Çünkü dengede Gibbs serbest enerji değişimi $\left(\Delta \mathrm{G}^{\circ}=0\right)$ sıfırdır ve adsorpsiyon ve desorpsiyon hızları birbirine eşittir. Böylece adsorban yüzeyine adsorplanan maddenin ve sulu çözeltideki adsorplanmadan kalan maddenin aktiviteleri oranı sabit kalır ve bu oran denge sabiti $\left(\mathrm{K}_{\mathrm{c}}\right)$ olarak adlandırılır. Bu sabit Eşitlik 2 ile hesaplanır (Atkins ve Paula, 2014).

$$
K_{c}=\frac{a_{s}}{a_{e}}=\frac{\gamma_{s} C_{s}}{\gamma_{e} C_{e}}
$$

Burada as ve ae sirasıyla; denge anında adsorban yüzeyine adsorplanan maddenin ve sulu çözeltideki adsorplanmadan kalan maddenin aktiviteleridir. $\gamma_{\mathrm{s}}$ ve $\gamma_{\mathrm{e}}$ ise sırasıyla denge anında adsorban yüzeyine adsorplanan maddenin ve sulu çözeltideki adsorplanmadan kalan maddenin aktivite katsayılarıdır. $C_{s}$ ve $C_{e}$ ise; denge anında adsorban yüzeyine adsorplanan maddenin ve sulu çözeltideki adsorplanmadan kalan maddenin konsantrasyonlarıdır. Aktivite katsayılarının yaklaşık aynı olduğu kabul edilirse,

$$
\mathrm{K}_{\mathrm{c}}=\frac{\mathrm{C}_{\mathrm{s}}}{\mathrm{C}_{\mathrm{e}}}
$$

Eşitlik 3 yazılabilir. Ayrıca, Van'tHoff eşitliği yazılırsa (Atkins ve Paula, 2014),

$$
\Delta G=\Delta G^{o}+R T \ln \left(K_{c}\right)
$$

Burada; $\Delta \mathrm{G}$, serbest enerji değişimi, $R$ ideal gaz sabiti $\left(8,314 \mathrm{~J} \cdot \mathrm{mol}^{-1} . \mathrm{K}^{-1}\right), \mathrm{T}$, mutlak sıcaklık $(\mathrm{K})$ ve $\mathrm{K}_{\mathrm{c}}$, denge sabitidir. $\Delta \mathrm{G}$ değeri denge anında sıfır olacağından, Eşitlik 5'e indirgenir.

$$
\Delta \mathrm{G}^{\mathrm{o}}=-\mathrm{RT} \ln \left(\mathrm{K}_{\mathrm{c}}\right)
$$

Bu eşitlik, adsorpsiyon prosesinin kendiliğinden gerçekleşip gerçekleşmeyeceğini tahmin etmek için kullanılan önemli bir eşitliktir. $\Delta \mathrm{G}^{\circ}$, adsorpsiyon prosesinin serbest enerji değişimidir.

Ayrıca, $\Delta \mathrm{G}^{\circ}$ için aşağıdaki termodinamik eşitlik te yazılabilir (Atkins ve Paula, 2014)

$$
\Delta G^{\mathrm{o}}=\Delta H^{\mathrm{o}}-T \Delta S^{\mathrm{o}}
$$

Eşitlik 5 ve $6^{\prime}$ dan,

$$
\ln \left(\mathrm{K}_{\mathrm{c}}\right)=-\frac{\Delta \mathrm{H}^{0}}{\mathrm{RT}}+\frac{\Delta \mathrm{S}^{0}}{\mathrm{R}}
$$

Eşitlik 7 elde edilir. Bu eşitlikten faydalanarak $\ln K_{c}$ ya karşı 1/T grafiği çizilirse (Şekil 2), eğimden $\Delta \mathrm{H}^{0}$ ve kesme noktasından $\Delta \mathrm{S}^{0}$ değerleri belirlenir. 


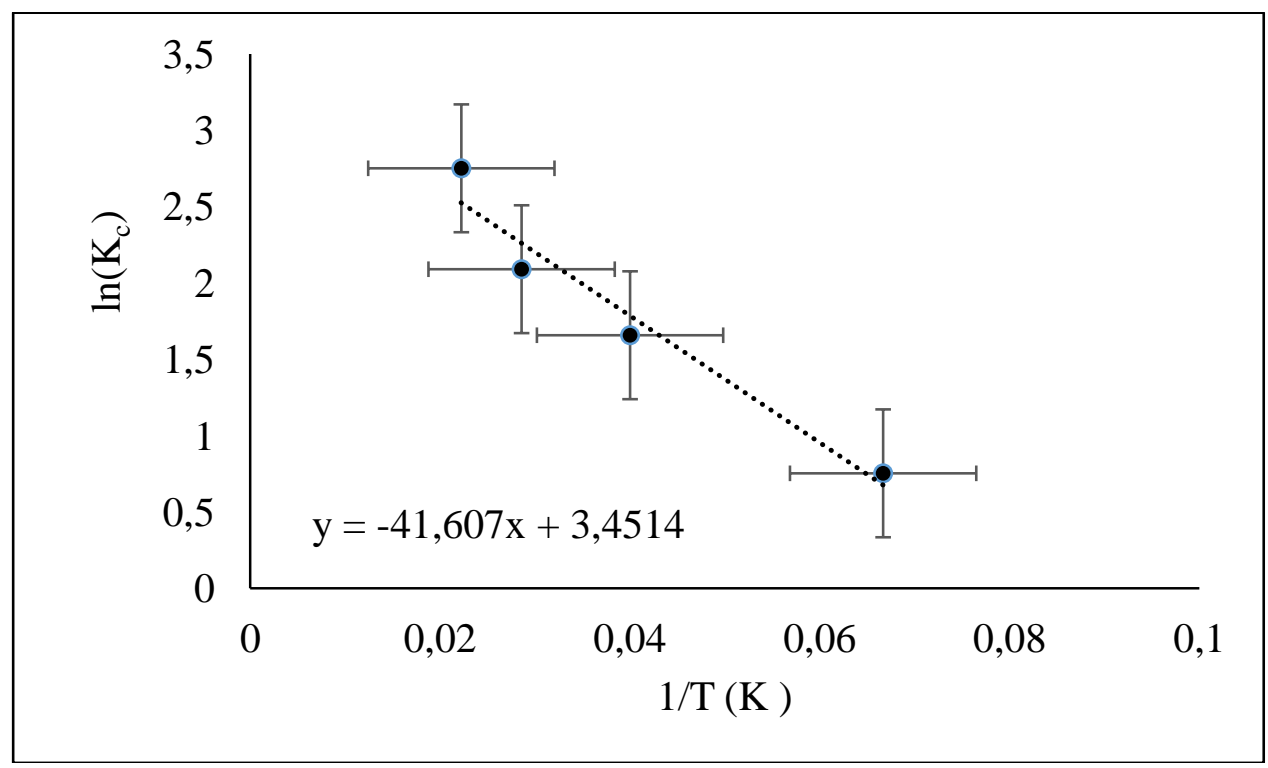

Şekil 2. $\ln \left(K_{c}\right)^{\prime}$ ye karşı 1/T grafiği

Figure 2. $\ln \left(K_{c}\right)$ vs. 1/T graph

Şekil 2'nin eğiminden $\Delta \mathrm{H}^{\circ}$, kesmesinden $\Delta \mathrm{S}^{\circ}$ ve Eşitlik 5'den $\Delta \mathrm{G}^{\circ}$ değerleri hesaplanmış ve sonuçlar Çizelge 3 'de verilmiştir.

Çizelge 3. Termodinamik Parametreler

Table 3. Thermodynamic Parameters

\begin{tabular}{|c|c|c|c|c|c|}
\hline$\Delta \mathrm{H}^{\circ}\left(\mathrm{kJ} \cdot \mathrm{mol}^{-1}\right)$ & $\Delta S^{\circ}\left(\mathrm{kJ} . \mathrm{mol}^{-1}\right)$ & \multicolumn{4}{|c|}{$\Delta \mathrm{G}^{\mathrm{o}}\left(\mathrm{kJ} \cdot \mathrm{mol}^{-1}\right)$} \\
\hline & & $288 \mathrm{~K}$ & $298 \mathrm{~K}$ & $308 \mathrm{~K}$ & $318 \mathrm{~K}$ \\
\hline 0,346 & 0,029 & $-7,92$ & $-8,20$ & $-8,49$ & $-8,78$ \\
\hline
\end{tabular}

$\Delta \mathrm{H}^{\circ}$ ve $\Delta \mathrm{S}^{\circ}$ değerlerinin pozitif çıkması ve $\Delta \mathrm{G}^{\circ}$ değerinin negatif çıkması nedeniyle adsorpsiyon prosesinin sırasıyla, endotermik, adsorban-adsorblanan arayüzeyinde düzensizlik artışı ve kendiliğinden gerçekleşen bir proses olduğunu gösterir. Sıcaklık arttıkça $\Delta G^{o^{\prime}}$ nin negatif değerinin artması, yüksek sıcaklıklarda perteknetat okso anyonunun aktif karbon üzerine adsorpsiyonuna olumlu etkide bulunduğunu gösterir. Benzer sonuçlar Mahmoud ve Seliman, 2014, çalışmasında da bulunmuştur.

\section{Adsorpsiyon İzosterik Isıs1(Isosteric Adsorption Heat)}

Sabit adsorblanan miktar için tanımlanan izosterik adsorpsiyon 1sısı $\left(\Delta \mathrm{Hx}, \mathrm{kJmol}^{-1}\right)$, adsorpsiyon prosesinin karakterizasyonu ve optimizasyonu tahmininde faydalı parametrelerden biri olup ClausiusClapeyron (Eşitlik 8) eşitliğine göre ln Ce'ye karşı 1/T grafiğinin eğiminden hesaplanır (Şekil 3) (Chowdhury ve diğ. 2011).

$$
\frac{\mathrm{d}\left(\ln \left(\mathrm{C}_{\mathrm{e}}\right)\right.}{\mathrm{dt}}=-\frac{\Delta \mathrm{Hx}}{\mathrm{RT}^{2}}
$$




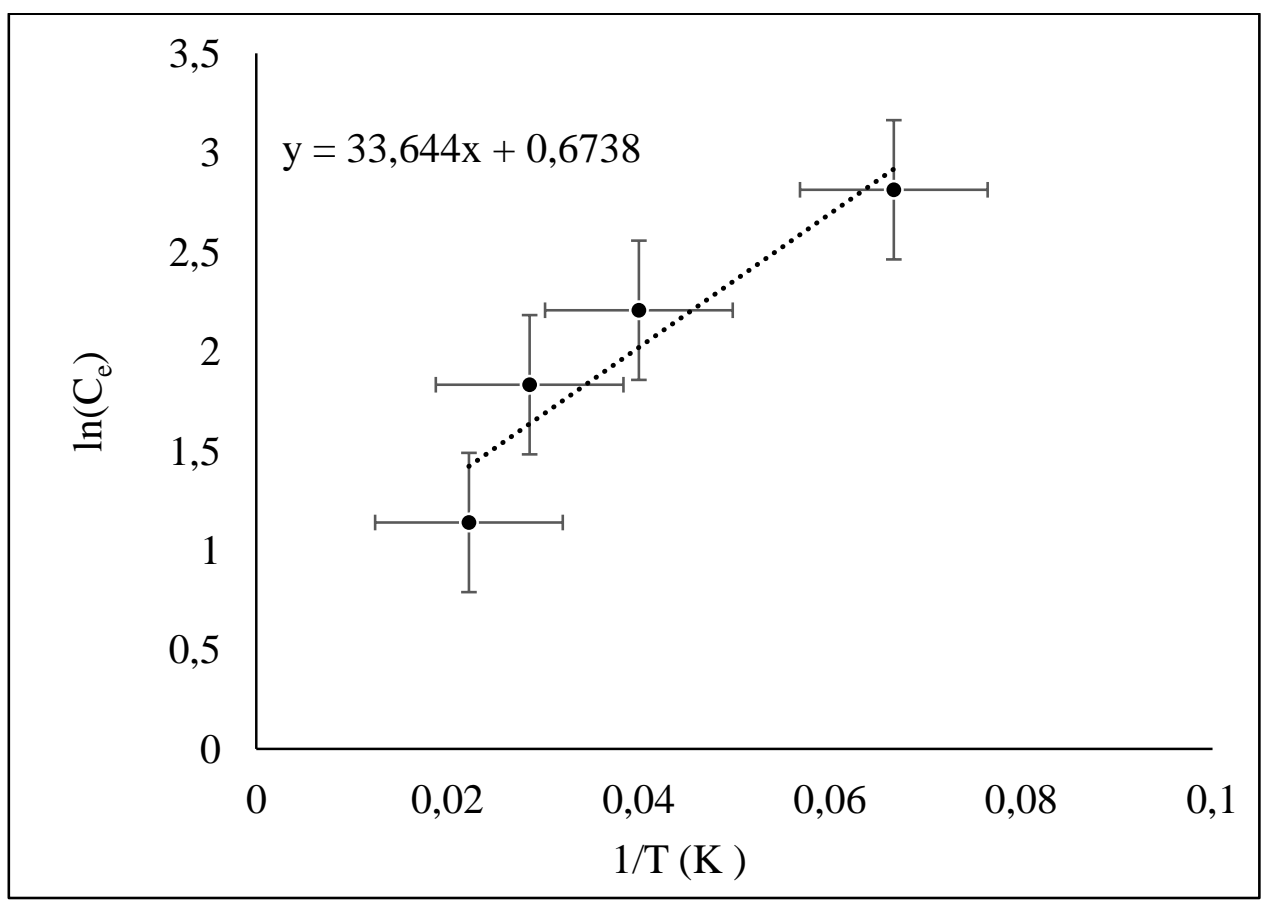

Şekil 3. $\ln \left(\mathrm{Ce}_{\mathrm{e}}\right)^{\prime}$ ye karşı 1/T grafiği

Figure 3. $\ln \left(C_{e}\right)$ vs. 1/T graph

Fiziksel adsorpsiyon için $\Delta \mathrm{Hx}, 80 \mathrm{~kJ} \cdot \mathrm{mol}^{-1}{ }^{\prime}$ den küçük ve kimyasal adsorpsiyon için ise 80-400 kJ.mol1 arasında değişmelidir (Doğan ve Alkan, 2003). Şekil 3'ün eğiminden $\Delta \mathrm{Hx}$ değeri $0,28 \mathrm{~kJ} \cdot \mathrm{mol}^{-1}$ olarak bulunduğundan adsorpsiyon prosesinin fiziksel adsorpsiyona uyduğu söylenebilir.

\section{Kendiliğinden Gerçekleşen Olaylarda Termodinamik Kriter(Thermodynamic Criteria for Spontaneity)}

Bir adsorpsiyon prosesinin termodinamik olarak uygun ve kendiliğinden olup olmadığ $\Delta \Delta \mathrm{G}^{\prime}$ in işaretine bakılarak belirlenebilir. Eğer $\Delta \mathrm{G}^{\circ}$ sıfırdan küçükse adsorpsiyon prosesi uygun ve kendiliğinden gerçekleşir. $\Delta \mathrm{G}^{0^{\prime}}$ in alacağı işaret, $\Delta \mathrm{H}^{\circ}$ ve $\Delta \mathrm{S}^{\circ}$ gibi termodinamik parametrelere bağlıdır. Bu durum aşağıdaki gibi açıklanabilir (Atkins ve Paula, 2014);

a) Eğer adsorpsiyon prosesi ekzotermik ise ( $\Delta \mathrm{H}^{\circ}$ negatif) ve katı sıvı ara yüzeyinde düzensizlik $\operatorname{artmış~(~} \Delta \mathrm{S}^{\circ}$ pozitif) ise, sıcaklık ne olursa olsun adsorpsiyon prosesi her zaman uygun ve kendiliğinden ( $\Delta \mathrm{G}^{\circ}$ negatif) dir

b) Eğer adsorpsiyon prosesi ekzotermik ise ( $\Delta \mathrm{H}^{\circ}$ negatif) ve katı sıvı ara yüzeyinde düzensizlik azalmış ( $\Delta \mathrm{S}^{\circ}$ negatif) ise, $\Delta \mathrm{H}^{\circ}$ değerinin $\mathrm{T} \Delta \mathrm{S}^{\mathrm{o}^{\prime}}$ dan büyük olması durumunda, adsorpsiyon prosesi uygun ve kendiliğinden ( $\Delta \mathrm{G}^{\circ}$ negatif) dir

c) Eğer adsorpsiyon prosesi endotermik ise ( $\Delta \mathrm{H}^{\circ}$ pozitif) ve katı sıvı ara yüzeyinde düzensizlik $\operatorname{artmış~}\left(\Delta \mathrm{S}^{\circ}\right.$ pozitif $)$ ise, $\Delta \mathrm{H}^{\circ}$ değerinin $\mathrm{T} \Delta \mathrm{S}^{\circ}$ den küçük olması durumunda, adsorpsiyon prosesi uygun ve kendiliğinden ( $\Delta \mathrm{G}^{\circ}$ negatif) dir

d) Eğer adsorpsiyon prosesi endotermik ( $\Delta \mathrm{H}^{\circ}$ pozitif) ise ve katı sıvı ara yüzeyinde düzensizlik azalmış ( $\Delta \mathrm{S}^{\circ}$ negatif $)$ ise, adsorpsiyon prosesi uygun ve kendiliğinden ( $\Delta \mathrm{G}^{\circ}$ negatif) gerçekleşeceği hiç bir sicaklik yoktur.

$\mathrm{Bu}$ açıklamalara göre adsorpsiyon prosesleri sadece belirli şartlarda kendiliğinden gerçekleşebilir. Adsorpsiyon proseslerinde uygun sicaklık aralığı, $\Delta G^{o^{\prime}}$ in sıfır olduğu sıcaklık (To) değerine göre belirlenir. Bu sıcaklık değeri, $\Delta \mathrm{G}^{0^{\prime}}$ a karşı T'nin grafik edilmesiyle bulunur (Şekil 4). Doğrunun kesme noktasından, $\Delta \mathrm{G}^{\prime}{ }^{\prime}$ n sıfır olduğu sıcaklık değeri bulunarak adsorpsiyon prosesinin uygun ve kendiliğinden gerçekleştiği aralık belirlenir. 


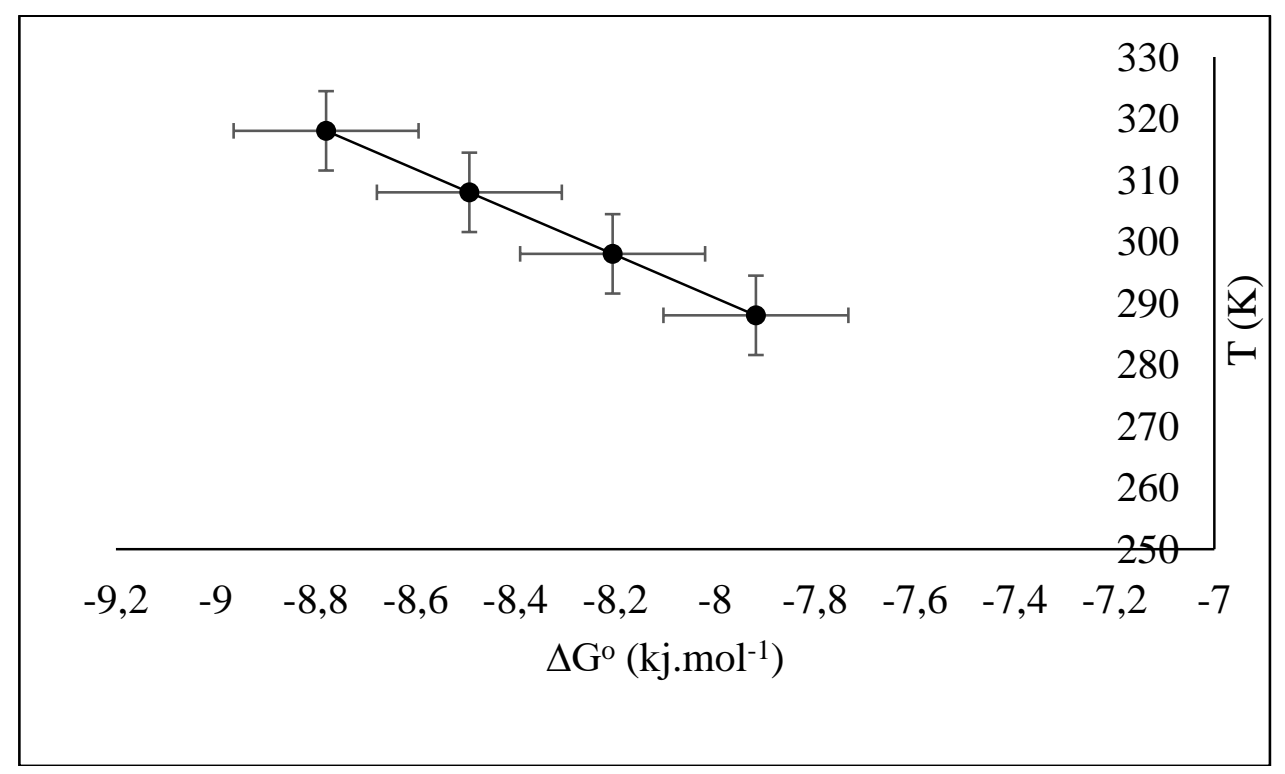

Şekil 4. $\Delta \mathrm{G}^{\prime}$ a karşı $\mathrm{T}$ grafiği

Figure 4. $\Delta G^{o}$ vs.T graph

Şekil 4'teki doğrunun kesme noktası olan $\Delta \mathrm{G}^{\mathrm{o}^{\prime}}$ in sıfır olduğu sıcaklık, $12 \mathrm{~K}$ olarak bulunmuştur. Bu değerin üzerindeki sıcaklıklarda, adsorblanan-adsorban ara yüzeyinde serbest enerjide daha fazla azalma olacağı için adsorpsiyon prosesi uygun ve kendiliğinden gerçekleşeceği söylenir. Özellikle adsorpsiyonun gerçekleşeceği sıcaklık aralığının bulunması, endüstriyel ölçekte optimum çalışma sıcaklığının belirlenmesinde önemli bir bilgi sağlamaktadır.

\section{SONUÇ (CONCLUSION)}

Bu çalışmada, aktif karbon üzerine radyoaktif perteknetat okso anyonunun adsorplanabilirliğini ve kendiliğinden gerçekleşip gerçekleşmeyeceğini tahmin edebilmek için termodinamik analizi incelenmiştir. Çizelge $2^{\prime}$ deki verilerden sıcaklığın artmasıyla adsorpsiyon kapasitesinin arttığ görülmektedir. Bununla birlikte, $\Delta \mathrm{H}^{\prime}$ in pozitif $\left(0,346 \mathrm{~kJ} \cdot \mathrm{mol}^{-1}\right)$ çıkması da adsorpsiyon prosesinin endotermik tepkimeyle yürüdüğünü göstermektedir. $\Delta S^{\circ}$ değerinin pozitif çıkması $\left(0,029 \mathrm{~kJ} \cdot \mathrm{mol}^{-1}\right)$, adsorban-adsorblanan ara yüzeyinde düzensizlik artışı olduğunu göstermektedir. $\Delta G^{\text {o }}$ değerlerinin negatif çıkması $\left(-7,92(288 \mathrm{~K})\right.$ ile $\left.-8,78(318 \mathrm{~K}) \mathrm{kJ} \cdot \mathrm{mol}^{-1}\right)$ ve $\Delta \mathrm{G}^{\mathrm{o}^{\prime}}$ in sıfır olduğu sıcaklığın $12 \mathrm{~K}$ olarak hesaplanması, adsorpsiyon prosesinin bu değerin üzerindeki her sıcaklıkta uygun ve kendiliğinden gerçekleşeceğini ifade etmektedir. $\Delta \mathrm{Hx}$ değeri, $0,28 \mathrm{~kJ} \cdot \mathrm{mol}^{-1}$ olarak bulunmuş ve $80 \mathrm{~kJ} \cdot \mathrm{mol}^{-{ }^{-1}} \mathrm{den}^{\mathrm{k}}$ üçük olduğu için adsorpsiyon prosesinin fiziksel olarak gerçekleştiği belirlenmiştir.

Sonuç olarak, belirli bir limitin üzerine çıkıldığında kansere neden olan radyoaktif teknesyumun aktif karbon üzerine adsorpsiyon termodinamik analizinin incelenmesi sonucu elde edilen bulguların endüstriyel çapta kurulacak bir tesis dizaynına katkıda bulunacağı söylenebilir.

\section{KAYNAKLAR (REFERENCES)}

Banavali, A.D., Raimondi, J.M., Moreno, E.M., McCurdy, D.E., 1995, "The determination of technetium-99 in low-level radioactive waste", Radioact. Radiochem., 6, 26-35.

Bastaban, B., 2014, "Teknesyum (99mTc) Elementinin Aktif Karbon Yardımıla Adsorbsiyonu" Atatürk Üniversitesi Fen Bilimleri Enstitüsü, Yüksek Lisans Tezi.

Bishop, M.E., Dong, H., Kukkadapu, R.K., Liu, C., Edelmann, R.E., 2011, “Bioreduction of Febearing clay minerals and their reactivity toward pertechnetate (Tc-99)", Geochim. Cosmochim. Acta, 75, 52295246 . 
Bors, J., Dultz, S., Riebe, B., 1999, "Retention of radionuclides by organophilic bentonite", Eng. Geol., 54, 195-206.

Chen, J., Veltkamp, J.C., 2002, "Pertechnetate removal by macroporous polymer impregnated with 2nitrophenyl octyl ether (NPOE)", Solvent Extr. Ion Exch., 20, 515-524.

Chowdhury, S., Mishra, R., Saha, P., \& Kushwaha, P., 2011, “Adsorption thermodynamics, kinetics and isosteric heat of adsorption of malachite green onto chemically modified rice husk", Desalination, 265(1-3), 159-168.

Del Cul, G.D., Bostick, W.D., Trotter, D.R., Osborne, P.E., 1993, “Technetium-99 removal from process solutions and contaminated groundwater", Sep. Sci. Technol., 28, 551-564.

Farrell, J., Bostick, W., Jarabek, R.J., Fiedor, J., 1999, “Electrosorption and reduction of pertechnetate by anodically polarized magnetite", Environ. Sci. Technol., 33, 1244-1249.

$\mathrm{Fu}, \mathrm{F} .$, Wang,Q., 2011, "Removal of heavy metal ions from wastewaters: a review", J. Environ.Manage, 92, 407-418.

HercigonjaRadmila V., Maksin Danijela D., Nastasovic'Aleksandra B., Trifunovic' Snežana S., Glodic'Pavle B., OnjiaAntonije E., 2012, “Adsorptive Removal of Technetium-99 Using Macroporous Poly (GMA-co-EGDMA) Modified with Diethylene Triamine", Journal of Applied Polymer Science, Vol. $123,1273-1282$.

Holm, E., Gäfvert, T., Lindahl, P., \&Roos, P. 2000, “In situ sorption of technetium using activated carbon", Applied Radiation and Isotopes, 53(1-2), 153-157.

Kumar, P.S., Senthamarai, C., Sai Deepthi, A.S.L., Bharani, R., 2013, “Adsorption isotherms, kinetics and mechanism of $\mathrm{Pb}$ (II) ions removal from aqueous solution using chemically modified agricultural waste", Can. J. Chem. Eng. 91, 1950-1956.

Kumar, S., Rawat, N., Kar, A.S., Tomar, B.S., Manchanda, V.K., 2011, "Effect of humic acid on sorption of technetium by alumina", J. Hazard. Mater., 192, 1040-1045.

León, M.G., 2005, "99Tc in the Environment: Sources, Distribution and Methods", J. Nucl. Radiochem. Sci. 6, 253-259.

Liang, L., Gu, B., Yin, X., 1996, “Removal of technetium-99 from contaminated ground water with sorbents and reductive materials", Sep. Technol. 6, 111-112.

Lieser, K.H., Bauscher, C.H., 1988, "Technetium in the hydrosphere and in the geosphere. II. Influence of $\mathrm{pH}$, of complexing agents and of some minerals on the sorption of technetium", Radiochim. Acta, 44, 125-128.

Dogan, M., Alkan, M., 2003, "Removal of methyl violet from aqueous solution by perlite", J. Colloid Interface Sci., 267, 32-41.

Mahmoud, M. R., Seliman, A. F. 2014, "Evaluation of silica/ferrocyanide composite as a dualfunction material for simultaneous removal of $137 \mathrm{Cs}+$ and $99 \mathrm{TcO} 4-$ from aqueous solutions", Applied Radiation and Isotopes, 91, 141-154.

Peter AtkinsandJulio de Paula, 2014, PhysicalChemistry 10th Edition Edition- 10th Edition oxforduniversitypress-newdelhi.

Pubchem,https://pubchem.ncbi.nlm.nih.gov/compound/SODIUM\%20PERTECHNETATE\#section=R elated-Compounds-with-Annotation.

Rout,T.K., Sengupta, D.K., Besra, L., 2006, "Flocculationimprovesuptake of 90Sr and 137Cs fromradioactiveeffluents", Int.J.Miner.Process., 79, 225-234.

Sangvanich,T., Sukwarotwat, V., Wiacek, R.J., Grudzien, R.M., Fryxell, G.E., Addle- man, R.S., Timchalk, C., Yantasee,W., 2010, "Selectivecapture of cesiumandthalliumfromnaturalwatersandsimulated wasteswithcopperferrocyanidefunctionalizedmesop oroussilica", J.Hazard.Mater.,182, 225-231.

Shakira, K., Ghoneimya, H.F., Hennawyb, I.T., Elkafrawyc, A.F., Beheira, S.G.E., Refaata, M., 2011, "Simultaneous removal of chromotrope $2 \mathrm{~B}$ and radionuclides from mixed radioactiveprocess wastewater using organo-bentonite", Eur. J. Chem., 2, 83-93. 
Shia Keliang, HouaXiaolin, Roos Per, Wu Wangsuo, 2012, “Determination of technetium-99 in environmental samples: A review", Analytica Chimica Acta, 709, 1- 20.

Suzuki, T., Fujii, Y., Yan,W., Mimura, H., Koyama, S., Ozawa, M., 2009, “Adsorption behavior of VII group elements on tertiary pyridine resin in hydrochloric acid solution", J. Radioanal. Nucl. Chem., 282, 641-644.

USEPA, 2002, “EPA facts about technetium-99”, https://www.epa.gov/radiation/radionuclide-basicstechnetium-99 